\title{
Effect of changes to Modified Look-Locker Inversion (MOLLI) sequence on T1 Mapping at 3.0 Tesla in healthy volunteers
}

\author{
Adam K McDiarmid ${ }^{1 *}$, David M Higgins², David P Ripley ${ }^{1}$, Akhlaque Uddin', Ananth Kidambi ${ }^{1}$ Peter P Swoboda', \\ Tarique A Musa', Bara Erhayiem', John P Greenwood ${ }^{1}$, Sven Plein ${ }^{1}$
}

From 17th Annual SCMR Scientific Sessions

New Orleans, LA, USA. 16-19 January 2014

\section{Background}

Diffuse myocardial fibrosis may be quantified with cardiovascular magnetic resonance (CMR) by calculating extra-cellular volume (ECV) from native and post-contrast $\mathrm{T} 1$ values. T1 values are affected by field strength and acquisition sequence. The Modified Look Locker Inversion Recovery (MOLLI) method of T1 mapping is a widely used approach, but many different acquisition schemes have been proposed. Flip angle and the effects of the prior inversions have both been suggested as potential targets for change in MOLLI scheme. In a 3,3,5 MOLLI scheme the third acquisition may be influenced by the two previous magnetisation inversions and incomplete recovery. 3,5 may be used as an alternative and performs similarly to $3,3,5$. However a 5,3 scheme offers more points unaffected by prior magnetisation and should maximise signal to noise (SNR) in native myocardium. The equation $\alpha \_S N R \max =\arccos (((\mathrm{T} 1-$ $\mathrm{T} 2)) /((\mathrm{T} 1+\mathrm{T} 2)))$ allows determination of flip angle for maximal signal in a given tissue for bSSFP readout, which is used by MOLLI. For myocardium this $\alpha$ SImax $=36^{\circ}$, where as blood pool signal is maximal at $50^{\circ}$. As signal is lower in native myocardium an appropriate flip angle should be selected to maximise this, before consideration of off-resonance effects on flip angle selection. Therefore we aimed to assess the effect of changes in MOLLI sequence on the accuracy of T1 mapping in healthy volunteers.

\footnotetext{
${ }^{1}$ Multidisciplinary Cardiovascular Research Centre \& The Division of Cardiovascular and Diabetes Research, Leeds Institute of Genetics, Health \& Therapeutics, University of Leeds, Leeds, West Yorkshire, UK Full list of author information is available at the end of the article
}

\section{Methods}

23 healthy volunteers were studied on a 3.0 Tesla (Philips Achieva TX) CMR system. Native T1 and 15 minute post-contrast (Gadovist $0.15 \mathrm{mmol} / \mathrm{kg}$ ) $\mathrm{T} 1$ maps were generated from mid-LV short axis with three different MOLLI protocols: 3,3,5 (flip angle 50 ${ }^{\circ}$ ); 3,3,5 (flip angle $35^{\circ}$ ); 5,3 (flip angle $35^{\circ}$ ). All acquisitions used minimum 3 -second pauses between sets of acquisitions. TR/TE/ voxel were $2.2 \mathrm{~ms} / 0.96 \mathrm{~ms} / 1.98 \times 1.98 \times 10 \mathrm{~mm}$. T1 measurements were made in the inter-ventricular septum. Means and standard deviations were compared between MOLLI T1 estimates and ECV calculated.

\section{Results}

23 volunteers (13 Male:10 Female; mean age $45 \pm$ 11 yrs; mean BSA $1.95 \pm 0.21$ ) T1 values and ECV were normally distributed and are as displayed below.

\section{Conclusions}

5,3 MOLLI acquisition resulted in higher native T1 value with lower standard deviation than $3,3,5$ scheme with either 35 or 45 flip angle. 5,3 reduces T1 underestimation associated with a traditional 3,3,5 MOLLI scheme. Subsequent calculated ECV has lower standard deviation. 5,3 MOLLI acquisition is shorter than 3,3,5,

Table 1

\begin{tabular}{lccc}
\hline & Native T1 ms (sd) & $\begin{array}{c}\text { T1 } 15 \text { min post } \\
\text { contrast ms }(\mathbf{s d})\end{array}$ & \% ECV (sd) \\
\hline 3,3,5 50 flip angle & $1113( \pm 69)$ & $484( \pm 59)$ & $26.0( \pm 5.3)$ \\
\hline 3,3,535 flip angle & $1158( \pm 70)$ & $494( \pm 49)$ & $28.0( \pm 5.7)$ \\
\hline 5,3 35 flip angle & $1205( \pm 36)$ & $503( \pm 58)$ & $25.6( \pm 3.0)$ \\
\hline
\end{tabular}


this may help minimise respiratory artefact in $\mathrm{T} 1$ and ECV maps when used in clinical examinations.

\section{Funding}

SP and JPG receive an educational research grant from Philips Healthcare. SP is funded by a British Heart Foundation fellowship

\section{Authors' details}

'Multidisciplinary Cardiovascular Research Centre \& The Division of Cardiovascular and Diabetes Research, Leeds Institute of Genetics, Health \& Therapeutics, University of Leeds, Leeds, West Yorkshire, UK. ${ }^{2}$ Philips Healthcare, Guildford, Surrey, UK.

Published: 16 January 2014

doi:10.1186/1532-429X-16-S1-P3

Cite this article as: McDiarmid et al:: Effect of changes to Modified Look-Locker Inversion (MOLLI) sequence on T1 Mapping at 3.0 Tesla in healthy volunteers. Journal of Cardiovascular Magnetic Resonance 201416 (Suppl 1):P3.

Submit your next manuscript to BioMed Central and take full advantage of:

- Convenient online submission

- Thorough peer review

- No space constraints or color figure charges

- Immediate publication on acceptance

- Inclusion in PubMed, CAS, Scopus and Google Scholar

- Research which is freely available for redistribution

Submit your manuscript at www.biomedcentral.com/submit
C Biomed Central 